\title{
Latest Results on Standard Model Higgs Boson at LEP
}

\author{
Shan JIN \\ University of Wisconsin-Madison, Madison, WI 53706, USA \\ Corresponding adress: CERN / EP division, CH-1211 Geneva 23, Switzerland \\ E-mail:Shan.Jin@cern.ch
}

\begin{abstract}
In this talk, the search results on Standard Model Higgs boson using LEP data collected at energies up to $209 \mathrm{GeV}$ are reported. An excess of $2.9 \sigma$ beyond the background expectation is found, consistent with the production of the Hoggs boson with a mass near $115 \mathrm{GeV} / \mathrm{c}^{2}$.
\end{abstract}

\section{INTRODUCTION}

In 2000 the four LEP experiments (ALEPH, DELPHI, L3, OPAL) have collected data at various energies between 200 and $209 \mathrm{GeV}$, for aprroximately $850 p b^{-1}$ (about $210 p b-1$ for each experiment), of which about $540 p b^{-1}$ above $206 \mathrm{GeV}$.

From combining the earlier data collected by the LEP experiments at center of mass energies up to $202 \mathrm{GeV}$, a $95 \%$ CL lower bound of $107.7 \mathrm{GeV} / \mathrm{c}^{2}$ on the mass of Standard Model (SM) Higgs boson has been obtained [1]. In this talk, we present an update of the SM Higgs search which includes the new data collected at center-of-mass enrgies uo to $209 \mathrm{GeV}$

At LEP the SM Higgs boson is expected to be produced mainly via Higgsstrahlung process $e^{+} e^{-} \rightarrow H Z$, while contributions from the $W W \rightarrow H$ fusion channel, $e^{+} e^{-} \rightarrow H \nu \bar{\nu}$, are typically below $10 \%$. The searches performed by the four LEP collaborations encompass the usual HZ final state topologies:

(1) Four-jet $(b \bar{b} q \bar{q})$ channel

(2) Missing energy $(b \bar{b} \nu \bar{\nu})$ channel

(3) Leptonic $\left(b \bar{b} e^{+} e^{-}\right.$and $\left.b \bar{b} \mu^{+} \mu^{-}\right)$channel

(4) Tau $\left(b \bar{b} \tau^{+} \tau^{-}\right.$and $\left.\tau^{+} \tau^{-} q \bar{q}\right)$ channel

In the four jet and tau channels, the overlap between the SM Higgs and MSSM Higgs analyses needs to be treated carefully in order not to subtract background twice since the same SM Higgs analyses are combined in the MSSM Higgs searches.

The analysis procedures of the four LEP experiments are described in individual documents [2]. 


\title{
LIKELIHOOD RATIO AND CONFIDENCE LEVELS
}

\author{
Estimator (test-statistic):
}

An Estimator (or a test-statistic) $X$ quantifies the "signal-ness" of an experiment. $X=-2 \ln Q$ where $Q=L(s+b) / L(b)$ is the likelihood ratio. Here the likelihood $L(s+b)$ assumes a signal process at Higgs mass $m_{H}$ in addition to the background process, and the likelihood $L(b)$ assumes no Higgs signal but background process only.

\section{Confidence Levels $C L_{s+b}$ and $C L_{b}$ :}

Given an observed value of the estimator $X_{\text {observed }}$, the confidence level on the signal + background and background - only hypotheses are calculated as $C L_{s+b}=$ $P_{s+b}\left(X \geq X_{\text {observed }}\right)$ and $C L_{b}=P_{b}\left(X \geq X_{\text {observed }}\right)$. The value $P_{b}$ is the estimator probability function for experiments with background process only, while $P_{s+b}$ is the estimator probability function for experiments with both background processes and a signal process of a given Higgs mass $m_{H}$ with the Standard Model cross section.

\section{Confidence Level $C L_{s}$ :}

The limit on $m_{H}$ is obtained via the limit on the Higgs cross section (number of signal events). So the confidence level $C L_{s}$ needs to be defined. Two methods are used in the $C L_{s}$ calculation in the LEP experiments:

(A) Generalized Bayesian Method [3]:

$C L_{s}=C L_{s+b} / C L_{b}$.

This method are used in DELPHI, L3, OPAL and LEP combined results.

(B) Signal Estimator Method [4]:

$C L_{s}=C L_{s+b}-\left(1-C L_{b}\right) \cdot e^{-S}$, where $S$ is the number of predicted signal events.

This method is used in the ALEPH results.

Both methods satisfy $C L_{s}=e^{-S}$ when zero event is observed, which is independent of background prediction. Method (B) gives typically about $0.5 \mathrm{GeV} / \mathrm{c}^{2}$ better sensitivity for the exclusion on $m_{H}$ than method (A).

\section{RESULTS}

Figure 1 shows $-2 \ln Q$ as a function of test Higgs mass. The solid line is the observation while the dashed / dash-dotted lines represent the median expected values for background / signal+background hypotheses. The dark- and light-shaded regions indicates the $\pm 1 \sigma$ and $\pm 2 \sigma$ bands around the background prediction. The 
expected curves and their spreads are obtained by replacing the observed data configuration by a large number of simulated event configuration.

A pronounced minimum at $m_{H}=115 \mathrm{GeV} / \mathrm{c}^{2}$ (maximum of the likelihood ratio Q) is observed, indicating a significant deviation from the background hypothesis. The minimun coincides with the signal+background expectation for a test mass of $115 \mathrm{GeV}$. Taking into account the asymmetric shape of the minimum, the observation is compatible with the SM Higgs boson production rate over the mass range from 114.6 to $115.8 \mathrm{GeV} / \mathrm{c}^{2}( \pm 1 \sigma)$.

Figure 2 shows $1-C L_{b}$ as a function of test Higgs mass, where the observation is given by the solid line while the expectations for the background and signal+background hypotheses are given by the dashed and dotted lines, respectively.

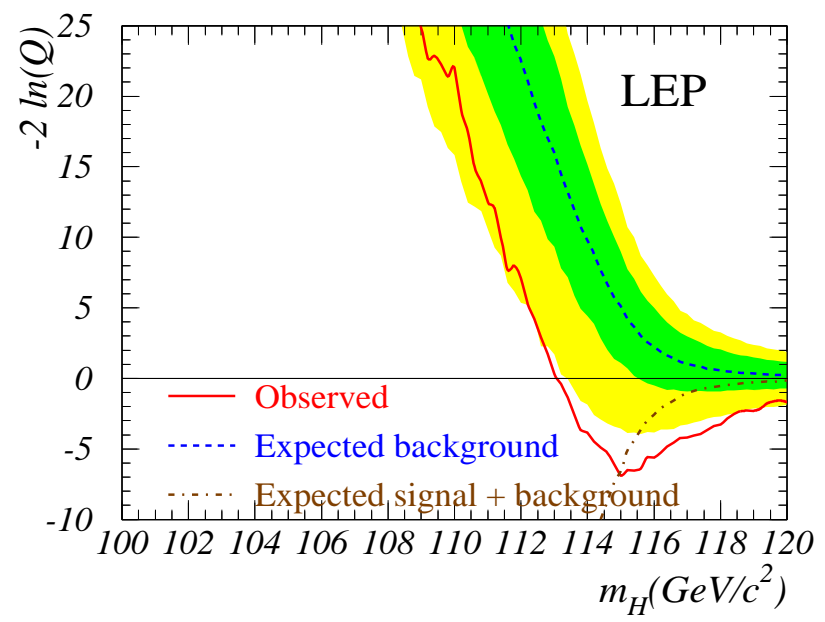

FIGURE 1. Likelihood Ratio $-2 \ln Q$ as a function of test Higgs mass $m_{H}$.

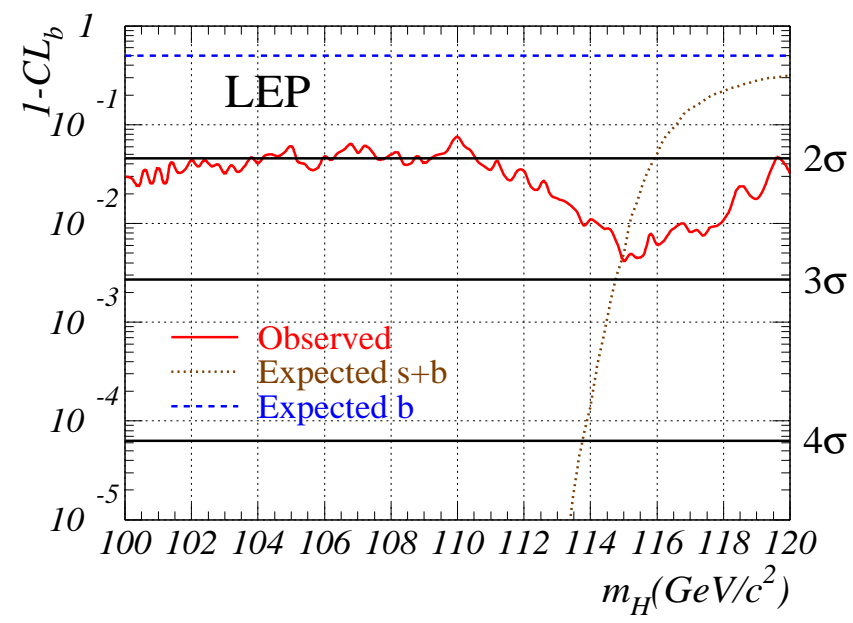

FIGURE 2. Probability $1-C L_{b}$ as a function of test Higgs mass $m_{H}$. The horizontal solid lines indicate deviations of 2- 3- and 4- $\sigma$ from the background hypothesis 
The minimum of $1-C L_{b}$ is $4.2 \times 10^{-3}$ at $115 \mathrm{GeV} / \mathrm{c}^{2}$, which corresponds to an excess of $2.9 \sigma$ beyond the background expectation.

A $95 \%$ confidence level lower limit on the SM Higgs mass may be set by identifying the mass region where $C L_{s}<0.05$. The median limit expected in the absence of a signal is $115.3 \mathrm{GeV} / \mathrm{c}^{2}$ and the limit observed by combining the LEP data is $113.5 \mathrm{GeV} / \mathrm{c}^{2}$.

\section{CONCLUSION}

An excess of $2.9 \sigma$ beyond the background expectation is observed in the combined SM Higgs search of four experiments at LEP using the data up to $209 \mathrm{GeV}$. The excess is consistent with the production of the Higgs boson with a mass near $115 \mathrm{GeV} / \mathrm{c}^{2}$. The measured lower bound for the mass of the SM Higgs boson is $113.5 \mathrm{GeV} / \mathrm{c}^{2}$.

\section{ACKNOWLEDGMENTS}

We wish to thank our colleagues from the accelerator divisions for the successful high energy operation of LEP. The results presented in this talk are due to the devoted work of many physicists from four LEP experiments. I am grateful to all of them. This work is supported in part by the United States Department of Energy through grand DE-FG-0295-ER40896.

\section{REFERENCES}

1. ALEP, DELPHI, L3 and OPAL Collab., The LEP Working group for Higgs boson searches, ALEPH 2000-028 CONF 2000-023, DELPHI 2000-050 CONF 365, L3 Note 2525, OPAL Technical Note TN-646.

2. ALEPH Collab., ALEPH 00-006 CONF 00-003;

DELPHI Collab., DELPHI 2000-092 CONF 391;

L3 Collab., L3 Note 2588;

OPAL Collab., OPAL Physics Note PN 426.

3. A.Read, CERN 2000-005.

4. S.Jin and P. McNamara, CERN 2000-005, physics/9812030. To be published in Nucl. Instrum. and Methods A. 